\title{
Anti-asthma and antitussive effects of a fermented extract of a mixture of Ramulus mori, Anthriscus sylvestris, and Salvia plebeian
}

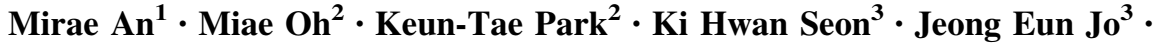 \\ Seong Kweon Lee ${ }^{3} \cdot$ Jeong-Keun Kim ${ }^{4} \cdot K_{\text {Kang Soon Shin }}{ }^{5} \cdot$ Jong-Ho Koh ${ }^{6}$. \\ Young-Hee Lim $^{2,7,8}$
}

Received: 31 March 2021/Revised: 14 June 2021/ Accepted: 22 July 2021/Published online: 10 August 2021

(C) The Korean Society of Food Science and Technology 2021

\begin{abstract}
Respiratory immunity is getting more important recently due to outbreak of respiratory diseases and increasing the concentration of fine dust. The aim of this study was to investigate respiratory protection effect of a fermented extract of medicinal plants (FEMP) containing Ramulus mori, Salvia plebeia, and Anthriscus sylvestris. The expression levels of $I L-8$ and $I L-17$ in LPS/poly-Larginine (PLA) and FEMP-cotreated A549 cells were lower than those in LPS/PLA only-treated cells. The levels of IgE, IL-17, and IL-4 in the bronchoalveolar lavage fluid (BALF) and serum of FEMP-treated mice with ovalbumin/
\end{abstract}

Young-Hee Lim

yhlim@korea.ac.kr

Mirae An

mran318@naver.com

Miae Oh

aldooh1234@naver.com

Keun-Tae Park

cerex@naver.com

Ki Hwan Seon

skh120701@hanmail.net

Jeong Eun Jo

whwjddms918@naver.com

Seong Kweon Lee

phdlsk1@naver.com

Jeong-Keun Kim

kjkim@kpu.ac.kr

Kwang Soon Shin

ksshin@kyonggi.ac.kr

Jong-Ho Koh

kohjh@kopo.ac.kr

1 Department of Public Health Science, Graduate School, Korea University, Seoul 02841, Republic of Korea
LPS-induced asthma were lower than the control levels. The lung inflammation score and the number of inflammatory cells in the BALF decreased by FEMP treatment. In the citric acid-induced coughing guinea pig, the FEMP treatment decreased the number of coughs. Therefore, FEMP shows anti-asthmatic and antitussive activities without hepatotoxicity and can be used as a compound aiming to improve respiratory health.

Keywords Anti-asthma Antitussive $\cdot$ Anti-inflammation . Fermented extract of medicinal plant

2 Department of Integrated Biomedical and Life Sciences, Graduate School, Korea University, Seoul 02841, Republic of Korea

3 R \& D Center, Biocean CO. LTD, Seoul 08591, Republic of Korea

4 Department of Chemical Engineering and Biotechnology, Korea Polytechnic University, Shihung-si, Gyeonggi-do 15073, Republic of Korea

5 Department of Food Science and Biotechnology, Kyonggi University, Suwon 16227, Republic of Korea

6 Department of Bio-Food Analysis and Processing, BioCampus Korea Polytechnic College, Nonsan 32943, Republic of Korea

7 School of Biosystems and Biomedical Sciences, College of Health Science, Korea University, Seoul 02841, Republic of Korea

8 Department of Laboratory Medicine, Korea University Guro Hospital, Seoul 08308, Republic of Korea 


\section{Introduction}

Respiratory immunity has recently become more important due to increases in the fine dust levels, and consequently, the incidences of respiratory diseases and outbreaks of novel respiratory diseases, such SARS-CoV, MERS-CoV, and COVID-19, are increasing. Fine dust, also known as particulate matter, is a mixture of particles and droplets with a diameter between 2.5 and $10 \mu \mathrm{m}$ (Brown et al., 2013) and is considered a global health issue. Fine dust can induce chronic inflammatory lung diseases, such as asthma, chronic obstructive pulmonary disease, extrinsic allergic alveolitis, and fibrosing alveolitis. In particular, asthma can be accelerated by the inhalation of fine dust, which can stimulate inflammation in the airway through Toll-like receptor-related signaling. Asthma is also triggered by remodeling airway conditions through aberrant DNA methylation and histone modifications, particularly among people who are exposed to fine dust in their early childhood. The incidences of respiratory diseases caused by novel viruses that induce severe lung damage by dysregulating the immune response and cytokine release syndrome are also increasing. Most respiratory diseases are similarly associated with inflammation caused by various reactions, such as the activation of neutrophils and eosinophils, an imbalance of $\mathrm{T}$ helper cells and oxidative stress (Wu et al., 2018). Consequently, the effects of the products or medicines for improving respiratory health are focused on anti-inflammation; for example, fluticasone, budesonide, mometasone, and beclomethasone, which are corticosteroids used to treat symptoms of respiratory diseases, have anti-inflammatory activities (Brightling et al., 2005; Leach et al., 1998). These medicines remarkably improve the symptoms of respiratory diseases but can cause unwanted side effects, such as adrenal suppression, osteoporosis, and diabetes (Phillipps, 1990). Therefore, effective and safe materials need to be developed for the prevention and improvement of respiratory diseases. Medicinal herbs that exert beneficial effects on humans without any side effects might be alternatives for those corticosteroid medicines.

Ramulus mori, the twig of Morus alba L. (Moraceae), exerts various beneficial effects on human health, such as antidiarrheic reactions, decreases in blood pressure, and antioxidant and anti-inflammatory activities ( $\mathrm{Yu}$ et al., 2019). Oxyresveratrol (trans-2,3',4,5'-tetrahydroxystilbene), the representative polyphenol in $R$. mori, is a hydroxy-stilbene with four hydroxyl groups, and has various health-promoting activities, such as anticancer effects and anti-allergic activity (Ashraf et al., 2015). Salvia plebeia R. Brown (Lamiaceae) is an annual or biennial plant belonging to the Labiatae family and serves as a folk remedy for diverse inflammatory diseases, such as hepatitis, influenza, and cough, in Asian countries (Harley et al., 2004). Rosmarinic acid, which is the representative active compound of $S$. plebeia $\mathrm{R}$. Brown, significantly inhibits the expression of cytokines and chemokines related to allergic inflammation, such as IL-4 and IL-5 (Sanbongi et al., 2004). Anthriscus sylvestris (Apiaceae), a biennial or shortlived perennial plant of the family Apiaceae, is commonly known as wild chervil or cow parsley and mainly grows in Europe, western Asia, and northwestern Africa. A. sylvestris has been traditionally used as a medicinal herb to obtain antitussive effects and for the treatment of asthma and headache (Fejes et al., 2000).

R. mori, S. plebeia R. Brown, and A. sylvestris exert beneficial effects independently on various inflammatory or allergic diseases (Jo et al., 2010; Zhang and Shi, 2010). The combination of medical herbs can produce synergistic effects compared with those observed with a single ingredient (Guimarães et al., 2011). In this study, a fermented extract of medicinal plants (FEMP) obtained from a mixture of R. mori, A. sylvestris, and S. plebeia R. Brown was prepared through bioconversion to enhance their medicinal effects, focusing on those on respiratory health. We examined the anti-inflammatory activity of the FEMP on cells from the human lung epithelium in vitro, and the antiasthma effect of the FEMP on the mouse model of OVA/ LPS-induced asthma and the antitussive activity of FEMP in a guinea pig animal model of CA-induced cough were investigated in vivo. Our study demonstrated that the FEMP exerted meaningful effects on respiratory health by inhibiting the expression of inflammatory cytokines and chemokines in experimental animals and human lung tissue cell lines and by showing antitussive activity.

\section{Materials and methods}

\section{Preparation of the fermented extract of medicinal plants (FEMP)}

Ramulus mori, Anthriscus sylvestris, and Salvia plebeia $\mathrm{R}$. Brown were purchased from Yong-woo Pharmacy Co (Seoul, Korea). The FEMP was prepared by fermentation of the water extract from a mixture of Ramulus mori, Anthriscus sylvestris, and Salvia plebeia R. Brown (w/w, 1:1:1). The conjugated enzyme cellulose prepared from Aspergillus niger (SUMYZYME-AC, Shin Nihon Chemical Co., Ltd., Anjo, Aichi, Japan) was added to the mixture with distilled water for bioconversion, and the mixture was then extracted at $45^{\circ} \mathrm{C}$ for $60 \mathrm{~h}$ and boiled at $90{ }^{\circ} \mathrm{C}$ for $30 \mathrm{~min}$ to stop enzyme activity. The fermented extract was filtered using filter paper (NO. 50, pore size: $12 \mu \mathrm{m}$, HDmicro, Seoul, Korea) and concentrated at $55^{\circ} \mathrm{C}$ using a 
rotary vacuum evaporator (N-1110, Eyela, Tokyo, Japan) until half of the filtrate remained. The concentrated extract was then lyophilized using a freeze dryer (PVTFD20, Ilshin Biobase, Dongduchen, Korea). The active compounds (oxyresveratrol and rosmarinic acid) in the FEMP were analyzed by high-performance liquid chromatography (HPLC) by using a YL9100 HPLC system (Younglin Instruments, Anyang-si, Korea) using a Luna-Pak ${ }^{\circledR} \mathrm{C}-18$ $(5 \mu \mathrm{m}, 4.6 \mathrm{~mm}$ i.d. $\times 250 \mathrm{~mm})$. The mobile phase was acetonitrile $\left(\mathrm{CH}_{3} \mathrm{CN}\right)$ and $10 \mathrm{mM} \mathrm{H} \mathrm{H}_{3} \mathrm{PO}_{4}(0 \mathrm{~min}, 10: 90$; 0-10 min, $25: 75 ; 10-30 \mathrm{~min}, 50: 50$, v/v). Flow rate was $0.7 \mathrm{~mL} / \mathrm{min}$, the injection volume was $100 \mu \mathrm{L}$ and the effluent was monitores at $325 \mathrm{~nm}$ with a photodiode array detector. Oxyresveratrol and rosmarinic acid were found at concentrations of $94 \mathrm{mg} / \mathrm{kg}$ and $12.144 \mathrm{~g} / \mathrm{kg}$ in the FEMP, respectively. The HPLC chromatograms of the active compounds are shown in Fig. S1. The FEMP used in this study was deposited under registration number FNCE01 in the R \& D Center, Biocean CO. LTD (Seoul, Korea).

\section{Cell culture}

The A549 cell line from the human lung was obtained from the Korean Cell Line Bank (Seoul, Korea). The cells were cultured in Roswell Park Memorial Institute (RPMI) 1640 containing $10 \%$ fetal bovine serum (FBS) and 1\% penicillin/streptomycin $(\mathrm{P} / \mathrm{S})$ (HyClone, Logan, UT, USA) at $37{ }^{\circ} \mathrm{C}$ in a humidified atmosphere with $5 \% \mathrm{CO}_{2}$. The culture medium was changed every $2 \mathrm{~d}$ during the incubation of the cells. The cells were seeded in a 96-well plate $\left(5 \times 10^{4}\right.$ cells $\left./ \mathrm{mL}\right)$ and in a six-well plate $\left(5 \times 10^{4}\right.$ cells $/$ $\mathrm{mL}$ ) and treated with various concentrations of the FEMP $(2.5,5$, and $10 \mu \mathrm{g} / \mathrm{mL})$.

\section{Cell viability assay}

The cell viability was measured using the 3-[4,5dimethylthiazol-yl]-2,5-diphenyltetrazolium bromide (MTT) (Ameresco, Solon, OH, USA) assay. A 549 cells were seeded at $5 \times 10^{4}$ cells $/ \mathrm{mL}$ in a 96-well plate, cultured for $24 \mathrm{~h}$, and treated with FEMP $(2.5,5$, and $10 \mu \mathrm{g} /$ $\mathrm{mL}$ ) for $24 \mathrm{~h}$. At the end of the incubation period, the medium was changed to RPMI containing MTT reagent, and the cells were incubated for $2 \mathrm{~h}$ at $37{ }^{\circ} \mathrm{C}$ in a humidified atmosphere with $5 \% \mathrm{CO}_{2}$. The formed formazan was extracted with $100 \mu \mathrm{L}$ of dimethyl sulfoxide (DMSO, Daejung, Siheung, Korea) for $30 \mathrm{~min}$ at room temperature, and the absorbance of each well at $540 \mathrm{~nm}$ was measured using a microplate reader (SpectraMax 340PC, Molecular Devices, San Jose, CA, USA). The relative cell viability (\%) was calculated by comparing the absorbance of the FEMP-treated cells with that of the negative control.

\section{Quantitative real-time polymerase chain reaction (qPCR)}

A 549 cells were seeded at $5 \times 10^{4}$ cells $/ \mathrm{mL}$ in a six-well plate and cultured for $24 \mathrm{~h}$ at $37^{\circ} \mathrm{C}$. The medium was then changed to RPMI 1640 without FBS for serum starvation for $24 \mathrm{~h}$. At the end of the incubation period, $\operatorname{FEMP}(2.5,5$, and $10 \mu \mathrm{g} / \mathrm{mL}$ ) and phosphate buffered saline (PBS, negative control) were added. After $1 \mathrm{~h}$, the cells were treated with lipopolysaccharide (LPS) and poly-L-arginine (PLA) (5 $\mu \mathrm{g} / \mathrm{mL}$ each) (Sigma-Aldrich, St. Louis, MO, USA) for $8 \mathrm{~h}$ to induce the expression of $I L-8$ and $I L-17$. The medium was removed by suction, and $1 \mathrm{~mL}$ of TRIzol reagent (Thermo Fisher Scientific) was added for the extraction of total RNA according to the manufacturer's manual. The total concentration of RNA was measured using a NanoDrop (ND-1000 spectrophotometer, Thermo Fisher Scientific). A RevertAid First Strand cDNA synthesis kit (Thermo Fisher Scientific) was used to convert the quantified RNA into cDNA, and the cDNA was used for qPCR using a KAPA SYBR FAST qPCR kit (KAPA Biosystems, Wilmington, NC, USA) and a QuantStudio 6 Flex system (Life Technologies, Carlsbad, CA, USA). The qPCR primers were obtained from Bioneer (Seoul, Korea), and their sequences used were as follows: $G A P D H$, forward $5^{\prime}$-ACC CAC TCC TCC ACC TTT GA- $3^{\prime}$, reverse $5^{\prime}$-CTG TTG CTG TAG CCA AAT TCG T- $3^{\prime} ; I L-8$, forward $5^{\prime}$ - CCA ACA CAG AAA TTA TTG TAA AGC- $3^{\prime}$, reverse $5^{\prime}$-TGA ATT CTC AGC CCT CTT CAA- $3^{\prime}$; and $I L-17$, forward $5^{\prime}$ CAC CTC ACC TTG GAA TCT C- $3^{\prime}$, reverse $5^{\prime}$-GGA TCT CTT GCT GGA TGG- $3^{\prime}$. The qPCR cycle consisted of preheating at $95{ }^{\circ} \mathrm{C}$ for $10 \mathrm{~min}$ followed by 40 cycles of $95{ }^{\circ} \mathrm{C}$ for $15 \mathrm{~s}, 60^{\circ} \mathrm{C}$ for $15 \mathrm{~s}$, and $72{ }^{\circ} \mathrm{C}$ for $30 \mathrm{~s}$. The relative gene expression was quantified using a comparative $\mathrm{Ct}$ method ( $\triangle \Delta \mathrm{Ct}$ method) (Livak and Schmittgen, 2001). GAPDH was used as the reference gene.

\section{Ovalbumin (OVA)/LPS-induced asthma model}

Five-week-old male BALB/c mice (18-20 g) were obtained from Orient Bio (Seongnam, Korea) and housed under the following conditions: $22 \pm 1{ }^{\circ} \mathrm{C}$, illumination intensity of $200 \pm 50 \mathrm{~lx}, 12 \mathrm{~h}$ light/12-h dark cycle and $50 \pm 5 \%$ relative humidity. The experimental protocols were approved by the Korea University Institutional Animal Care and Use Committee (Approval No. KUIACUC2019-0026). All the mice had free access to their diet (No. 5053, LABDIET, St. Louis, MO, USA) and water. After acclimation for one week, the mice were randomly divided into four groups ( 8 mice per group): the mice in group 1 (normal control; Nor group) were treated with vehicle (PBS) and were not subjected to OVA/LPS sensitization and challenge, the mice in group 2 (OVA/LPS-induced 
asthma control; Model group) were treated with vehicle and subjected to OVA/LPS sensitization and challenge, the mice in group 3 (low FEMP concentration; FEMP50 group) were treated with $50 \mathrm{mg} / \mathrm{kg} /$ day FEMP and were subjected to OVA/LPS sensitization and challenge, and the mice in group 4 (high FEMP concentration; FEMP100 group) were treated with $100 \mathrm{mg} / \mathrm{kg} / \mathrm{day}$ FEMP and subjected to OVA/LPS sensitization and challenge. PBS and the FEMP were orally administered for one week (14-21 days, daily), and the body weight was measured weekly. To induce asthma, the mice were subjected to sensitization and challenge with antigens, namely, OVA (Sigma-Aldrich) and LPS. OVA/LPS sensitization was conducted on days 0 and 7 through the intraperitoneal injection of $0.2 \mathrm{~mL}$ of a mixture of $75 \mu \mathrm{g}$ of OVA, $50 \mu \mathrm{g}$ of LPS, and $2 \mathrm{mg}$ of aluminum hydroxide (Sigma-Aldrich). OVA/LPS challenge was performed on days 14, 15, and 21 via the inhalation of $1 \%$ OVA/LPS for 30 min per day using a nebulizer (NE-C28, Omron, Tokyo, Japan) (Fig. S2). The normal group was exposed to PBS under the same conditions.

\section{Citric acid (CA)-induced cough model}

Male Hartley guinea pigs (350-400 g) were obtained from Koatech (Pyeongtaek, Korea) and were housed under the following conditions: $24 \pm 1{ }^{\circ} \mathrm{C}, 12$-h light/12-h dark cycle and $50 \pm 5 \%$ relative humidity. The experimental protocols were designed in accordance to the guidelines of the Ethics Committee for the Use of Experimental Animals at Kyonggi University (Approval No. 2019-013). All the guinea pigs had free access to their diet (Purina Guinea pig diets, Purina, Seongnam, Korea) and water. After acclimation for one week, the guinea pigs were randomly separated into four groups (8 guinea pigs per group): group 1 (normal control; Nor group) was treated with vehicle (PBS) and was not subjected to stimulation with CA (SigmaAldrich), group 2 (CA-induced cough control; Model group) was treated with vehicle and stimulated with CA, group 3 (low FEMP concentration; FEMP50 group) was treated with FEMP at a dose of $50 \mathrm{mg} / \mathrm{kg} /$ day and stimulated with CA, and group 4 (high FEMP concentration; FEMP100 group) was treated with $100 \mathrm{mg} / \mathrm{kg} /$ day FEMP and stimulated with CA. The vehicle and FEMP were orally administered for one week (0-7 days, daily). One hour after the last administration, the guinea pigs were exposed to a nebulizer filled with $0.2 \mathrm{M}$ citric acid for 10 min to induce coughing, and the number of coughs over the 15-min period immediately following the treatment was counted (Fig. S3).

\section{Serum biochemical analysis}

The animals were fasted for $24 \mathrm{~h}$ before sacrifice under anesthesia, which was administered via the inhalation of isoflurane $(0.25-4 \%)$. Blood was collected in a serum separator tube (SST) by cardiac puncture and centrifuged for $10 \mathrm{~min}$ at $3000 \times g$ to separate the serum. The serum levels of glutamic oxaloacetic transaminase (GOT) and glutamic pyruvic transaminase (GPT) were measured using FUJI DRY-CHEM slides (FUJIFILM Co., Tokyo, Japan).

\section{Bronchoalveolar lavage fluid (BALF) collection and cell counting}

BALF collection was performed immediately after blood collection by inserting a catheter into the trachea. Cold PBS ( $1 \mathrm{~mL})$ was injected using a syringe into the lungs of mice and sucked three times to obtain the BALF. The BALF was centrifuged at $400 \times g$ and $4{ }^{\circ} \mathrm{C}$ for $10 \mathrm{~min}$, and the supernatant was separated and stored at $-80{ }^{\circ} \mathrm{C}$ for cytokine and immunoglobulin measurements. The cell pellets were resuspended in $200 \mu \mathrm{L}$ of PBS and centrifuged at $400 \times g$ and $4{ }^{\circ} \mathrm{C}$ for $10 \mathrm{~min}$. The supernatant was discarded, and the pellets were resuspended in $200 \mu \mathrm{L}$ of ACK lysis buffer $\left(\mathrm{NH}_{4} \mathrm{Cl} 150 \mathrm{mM}, \mathrm{KHCO}_{3} 10 \mathrm{mM}\right.$, and $\mathrm{Na}_{2}$ EDTA $0.1 \mathrm{mM}, \mathrm{pH} 7.3$ ) and maintained at $4{ }^{\circ} \mathrm{C}$ for $10 \mathrm{~min}$ for the lysis of red blood cells. After lysis, $800 \mu \mathrm{L}$ of PBS was added to stop the reaction, and the mixture was centrifuged at $400 \times g$ and $4{ }^{\circ} \mathrm{C}$ for $10 \mathrm{~min}$. The cell pellets were resuspended in $200 \mu \mathrm{L}$ of PBS, and the total cell count was determined using a hemocytometer under a light microscope (CKX41, Olympus, Tokyo, Japan). A moderate amount of cell suspension was concentrated using a cytocentrifuge (Hanil, Daejeon, Korea) at $12,000 \mathrm{rpm}$ for $10 \mathrm{~min}$. Subsequently, the cytospin smears were stained with Diff-quick stain according to the manufacture's instruction (Sysmex, Kobe, Japan), and the inflammatory cells were observed through a light microscope. Stained immune cells were distinguished by their characteristic staining patterns. Neutrophils are about $12-15 \mu \mathrm{m}$ and the nucleus, cytoplasm, and granules are blue, pink, and violet, respectively. Eosinophils are about $12-17 \mu \mathrm{m}$ and the nucleus, cytoplasm, and granules are blue, blue, and red, respectively. Lymphocytes are 9-12 $\mu \mathrm{m}$ and the nucleus is purple/dark blue and the cytoplasm shows typical basophilic characteristics such as thin border containing red granules. Macrophages are $19-22 \mu \mathrm{m}$ and the nucleus is violet and the cytoplasm is light blue. At least 100 cells were counted randomly. 


\section{Histological analysis}

The lungs and livers of the mice were removed, immediately fixed with $10 \%$ formalin and then embedded in paraffin. The embedded tissues were sectioned at approximately 3-5 $\mu \mathrm{m}$ thickness, dewaxed in xylene, dehydrated in 70, 80, 90 and $100 \%$ ethanol, and then stained with hematoxylin and eosin (H\&E). The degree of pathological changes in the lung was scored according to the Table S1 (Dubin and Kolls, 2007), ( Bayes et al., 2016).

\section{Quantification of cytokines and immunoglobulin in the BALF and serum}

The mouse serum and BALF were used to assess the levels of IgE, IL-17, and IL-4 using an IgE mouse uncoated ELISA kit with plates (Invitrogen, Carlsbad, CA, USA), an IL-17 mouse ELISA kit (Invitrogen), and an IL-4 mouse ELISA kit (Invitrogen), respectively, according to the protocols recommended by the manufacturer. After the reaction, the absorbance at $450 \mathrm{~nm}$ was measured using a microplate reader (SpectraMax 340PC, Molecular Devices), and the concentrations of IgE, IL-17, and IL-4 were calculated according to the corresponding standard curve.

\section{Statistical analysis}

The significance of the differences between the results obtained for the various groups was evaluated through oneway analysis of variance (ANOVA) using the Statistical Package for the Social Science (SPSS) statistical analysis program version 25 . The in vitro and in vivo results are expressed as the mean \pm standard deviation (SD) and the mean \pm standard error of the mean (SEM), respectively. A $p$ value was less than 0.05 was considered to indicate a significant difference, and post hoc tests were performed using Tukey's honestly significant difference (HSD) method.

\section{Results and discussion}

\section{Effect of FEMP on cell viability}

A549 cells were used to evaluate the anti-inflammatory effect of the FEMP in vitro. Compared with that of the negative control group, cell viabilities show that the concentrations of FEMP used in this study did not exhibit any cytotoxicity (Fig. S4).

\section{Reducing effect of FEMP on the cytokines gene expression level in cells treated with LPS/PLA}

LPS is commonly found in the environment and can be a crucial asthma-aggravating factor in the case of its absorption (Zhang et al., 2016). PLA can imitate the function of major basic proteins that cause airway hyperresponsiveness by stimulating airway myocytes (Duan et al., 2004) and induce an inflammatory pathology by injuring the cell membrane (Zhang et al., 2016). Therefore, A549 cells were cotreated with LPS and PLA to induce inflammation in this study, and the effect of FEMP on the expression of genes related to inflammatory cytokines in A549 cells was assessed by qPCR. IL-4 is a main factor that has a significant impact on asthma. In the case of LPSinduced cell experiments, we analyzed IL- 8 that affects to asthma related to inflammation instead of IL-4 because LPS-induced cell model has limitations in inducing IL-4 that is secreted by basophilic granulocytes and eosinophils (Sokol et al., 2008) and then induces the differentiation of naïve helper T cells to Th2 cells that produce IL-4, and IL4 subsequently acts as an autocrine. IL- 8 is a chemokine produced by airway epithelial cells and smooth muscle cells, and its main function is chemotaxis to activate neutrophils and eosinophils (Hedges et al., 2000). IL-17 is a proinflammatory cytokine produced by $\mathrm{T}$ helper 17 cells, which are generated by cytokines such as IL-6, IL- $1 \beta$, IL23 , and TGF- $\beta$ (Kolls and Lindén, 2004). TNF- $\alpha$ and IL-1 $\beta$ are typical proinflammatory cytokines that stimulate inflammatory responses and they show increased circulation in the patients with asthma (Berry et al., 2007; Lambrecht et al., 2019). The $I L-8$ expression level in the cells treated with LPS/PLA alone was 4.7-fold higher than that of the negative control (Fig. 1A). The IL-8 expression levels in the cells cotreated with LPS/PLA and FEMP (2.5, 5 , and $10 \mu \mathrm{g} / \mathrm{mL}$ ) significantly decreased compared with the cells treated with LPS/PLA alone in a dose-dependent manner. The $I L-17$ expression level in the cells treated with LPS/PLA alone was 1.9-fold higher than that of the negative control (Fig. 1B). The $I L-17$ expression levels in the cells cotreated with LPS/PLA and FEMP (2.5, 5, and $10 \mu \mathrm{g} / \mathrm{mL}$ ) significantly decreased in a dose dependent manner compared with the cells treated with LPS/PLA alone. Expression level of $T N F-\alpha$ in the cells treated with LPS/PLA alone was 1.9-fold higher than that of the negative control (Fig. 1C). Although it was not significant, expression level of $T N F-\alpha$ decreased in the cells cotreated with LPS/PLA and FEMP compared with the cells treated with LPS/PLA alone. Expression level of $I L-1 \beta$ in the cells treated with LPS/PLA alone was 1.5 -fold higher than that of the negative control, but it was not significant (Fig. 1D). Unexpectedly, although it was not significant, expression level of $I L-1 \beta$ in the cells cotreated with LPS/PLA and 
A

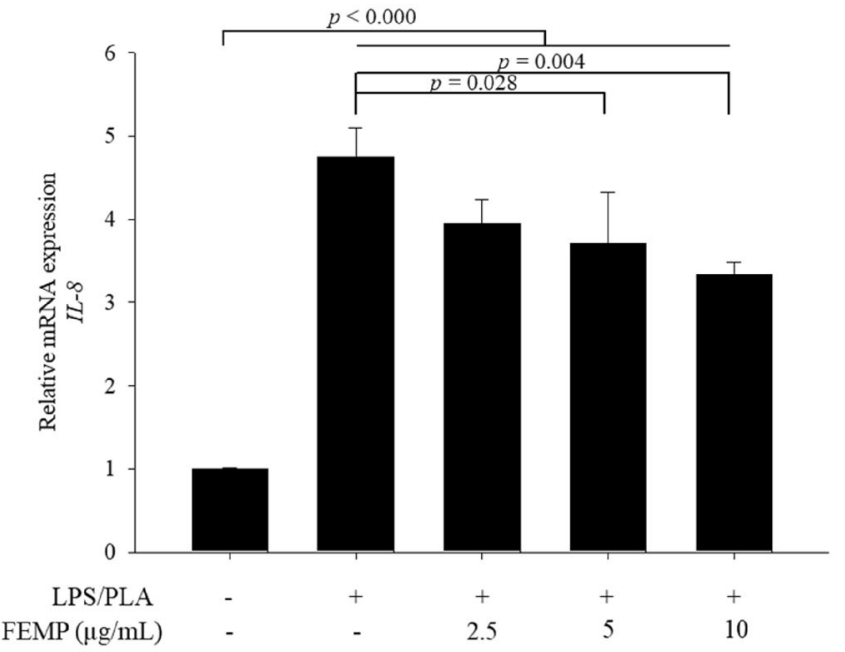

C

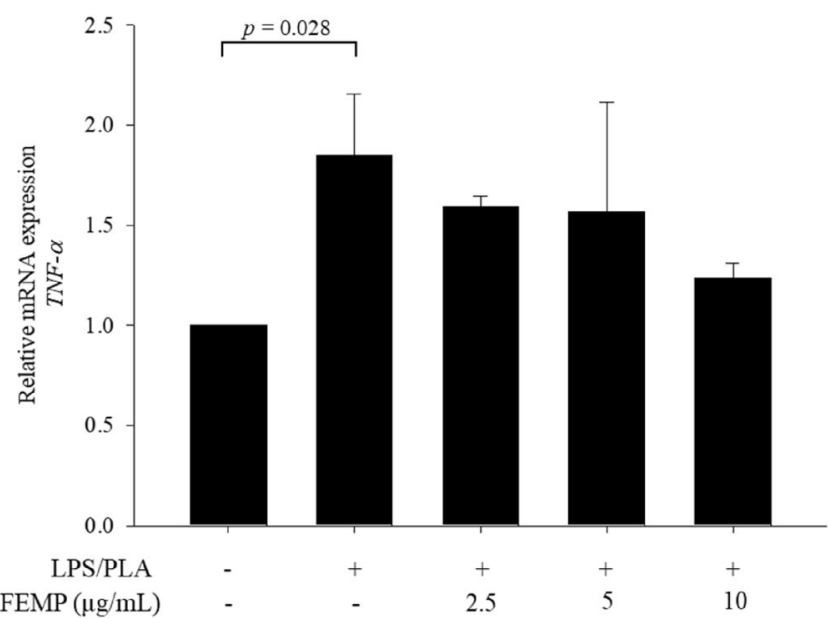

Fig. 1 The mRNA expression levels related to inflammatory cytokines decreased by FEMP treatment. A549 cells $\left(5 \times 10^{4}\right.$ cells/ $\mathrm{mL}$ ) exhibited inflammation induced by LPS/PLA and were treated with the FEMP and then the mRNA expression levels of $I L-8(\mathbf{A}), I L$ -

FEMP $(2.5 \mu \mathrm{g} / \mathrm{mL})$ increased compared with the cells treated with LPS/PLA alone, however, expression levels of $I L-1 \beta$ in the cells cotreated with LPS/PLA and FEMP $(10 \mu \mathrm{g} / \mathrm{mL})$ significantly decreased compared with the cells cotreated with LPS/PLA and FEMP $(2.5 \mu \mathrm{g} / \mathrm{mL})$. These results show that the LPS/PLA-induced expression of inflammatory cytokines genes was significantly reduced by treatment with the FEMP in a dose-dependent manner, which suggests that the FEMP exerts anti-inflammatory effects in lung tissue.
B

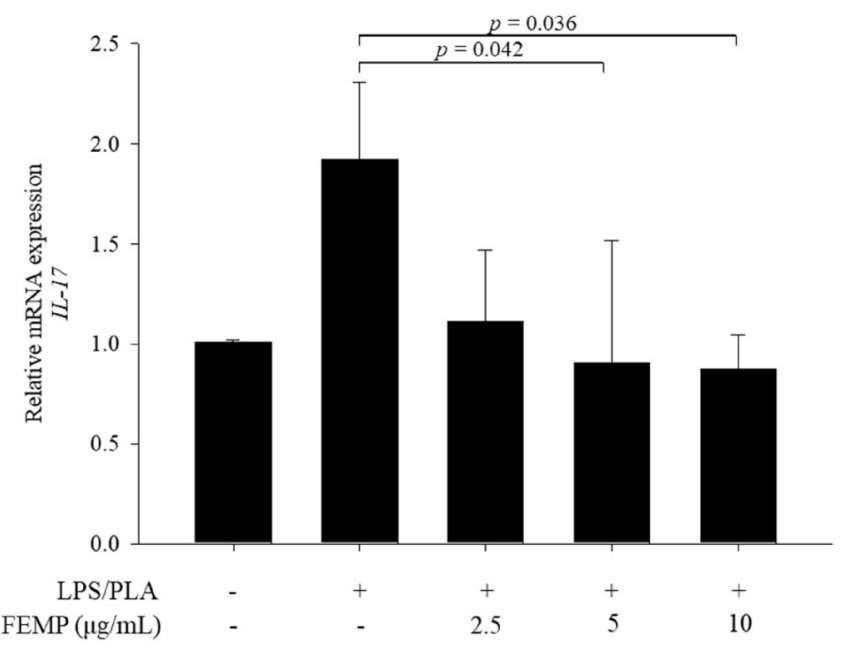

D

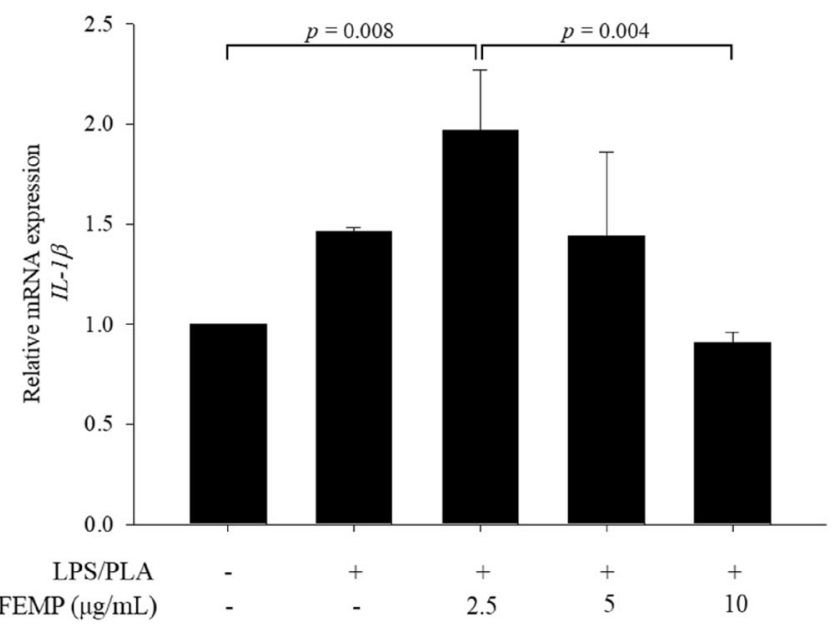

$17(\mathbf{B}), T N F-\alpha(\mathbf{C})$, and $I L-1 \beta(\mathbf{D})$ were measured by qPCR. The data are expressed as the mean \pm SD and were analyzed by one-way ANOVA/Tukey's HSD method

Effect of FEMP on the body weight change and hepatotoxicity in OVA/LPS-induced asthma mice

To evaluate the anti-inflammatory effect of FEMP in vivo, we used an OVA/LPS-induced asthma mouse model. To induce asthma, OVA is commonly used for whole-body sensitization, and the co-administration of OVA with LPS is also used to induce asthma and the corresponding immune response in studies of bronchial asthma pathogenesis. During the first two weeks after sensitization, the body weights of the mice with OVA/LPS-induced asthma, regardless of whether the mice were orally administered the FEMP, were lower than those of the mice belonging to the normal group; however, no significant difference in 
body weight was found among the groups, including the normal group, at the end of the experiment (Fig. 2A). In addition, the serum GOT and GPT levels did not show a significant difference among the groups (Table 1). To investigate the histological changes in liver tissue, $\mathrm{H} \& \mathrm{E}$ staining was performed, and no differences in tissue morphology were detected (Fig. 2B). The results suggest that the oral administration of the FEMP within the concentration range used in this study did not result in hepatotoxicity.

\section{Suppression of secreting immunoglobulin $\mathrm{E}$ and inflammatory cytokines by FEMP in OVA/LPS- induced asthma mice model}

IgE plays a vital role in type I hypersensitivity, such as allergic asthma, allergic rhinitis and atopic dermatitis (Gould et al., 2003). IL-4, which, similarly to IL-17, is a proinflammatory cytokine, stimulates the activation of $\mathrm{B}$ cells, $\mathrm{T}$ cells and naïve $\mathrm{T}$ helper cells, and the overproduction of IL-4 is also related to allergic diseases, which are caused by disproportionate immune responses involving $\mathrm{T}$ helper 2 cells and allergens (Steinke and Borish, 2001). Therefore, IgE, IL-4, and IL-17 are the major factors responsible for the induction of allergic diseases, including asthma, by activating $\mathrm{T}$ helper cells and leading to the secretion of other inflammatory cytokines (Wong et al., 2001). To investigate the anti-asthmatic effect of the FEMP on OVA/LPS-induced asthmatic mice, the expression levels of IgE and cytokines (IL-4 and IL-17) in the
BALF and serum were measured by ELISA. The secretion of IgE in both the BALF and serum was significantly higher in the model group than in the normal group, whereas the secretion of IgE in the BALF and serum was significantly decreased in the FEMP50 and FEMP100 groups, respectively, compared with the model group (Fig. 3A). No significant difference in the secretion of IL17 in serum was found among the groups, regardless of FEMP treatment, whereas the secretion of IL-17 in the BALF was significantly lower in the FEMP100 group than in the model group (Fig. 3B). The secretion of IL-4 in the BALF was significantly increased in the model group compared with the normal group, whereas that in the FEMP100 group was significantly lower than that in the model group (Fig. 3C). No IL-4 secretion was detected in serum. Both IL-4 and IL-17 are proinflammatory cytokines that are produced in response to physical triggers of the immune response, such as infections, toxins, medications, and allergens. Once antigen-presenting cells are stimulated, naïve $\mathrm{T}$ cells undergo differentiation into various $\mathrm{T}$ helper subsets according to the trigger of the immune response. In the case of allergen triggers, naïve $\mathrm{T}$ cells are mostly stimulated to differentiate into $\mathrm{T}$ helper 2 cells that secrete IL-4 and IL-2, and helper T2 cell-induced inflammation increases IgE synthesis, airway hyperresponsiveness, and mucus hypersecretion in the airway (Heuer et al., 2013). IL-17 is secreted by T helper 17 cells that are differentiated in response to infections or autoimmunity. Recent studies revealed that IL-17 contributes to the development of severe asthma (Ramakrishnan et al., 2019). The secretion
A

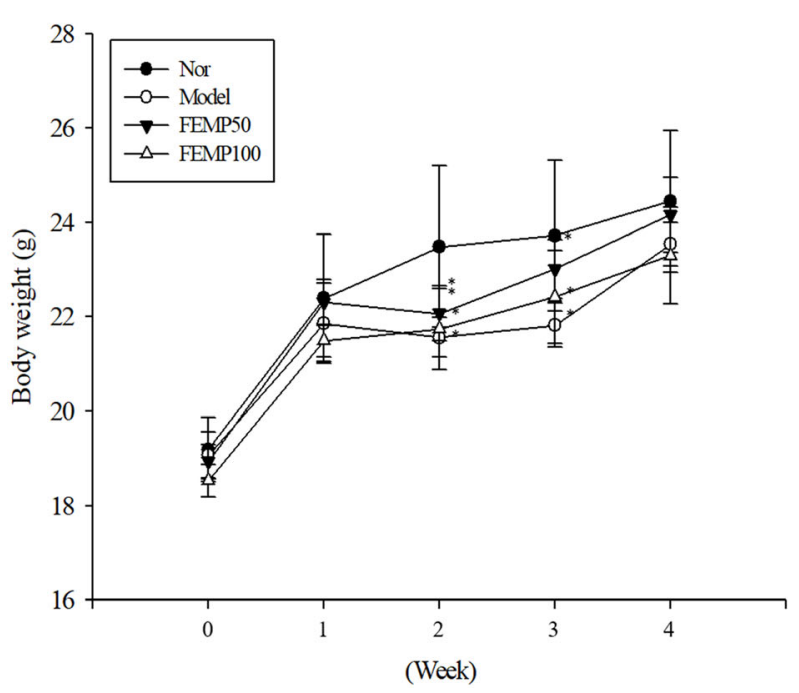

Fig. 2 Effect of the FEMP on the body weight changes (A) and liver tissue morphology (B) in OVA/LPS-induced asthma mice ( $\mathrm{n}=8$ /group). Nor, normal control; Model, OVA/LPS-induced asthma control; FEMP50, OVA/LPS-induced asthmatic mice treated with $50 \mathrm{mg} / \mathrm{kg} /$ day FEMP; FEMP100, OVA/LPS-induced asthmatic

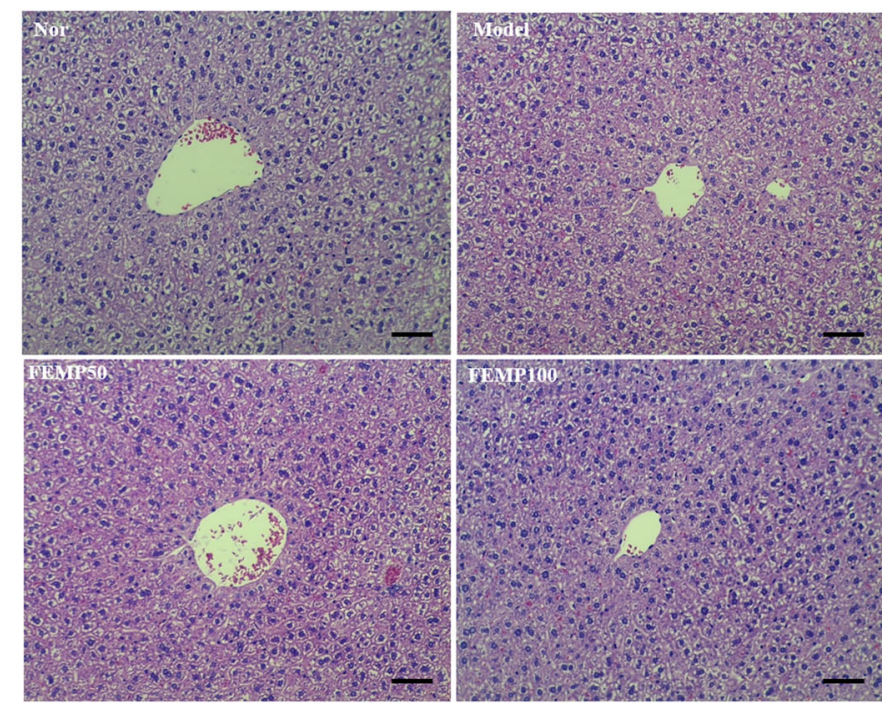

mice treated with $100 \mathrm{mg} / \mathrm{kg} /$ day FEMP. The data are expressed as the mean \pm SEM and were analyzed by one-way ANOVA/Tukey's HSD method. Significant differences are marked with asterisks: $* p<0.05$ and $* * p<0.01$. The scale bar indicates $50 \mu \mathrm{m}$, and the tissue was observed at $200 \times$ magnification 
Table 1 Effect of the FEMP on the serum GOT and GPT levels in mice with OVA/LPS-induced asthma $(\mathrm{n}=8 /$ group $)$

\begin{tabular}{lllll}
\hline & Nor & Model & FEMP50 & FEMP100 \\
\hline GOT (U/L) & $76.2 \pm 14.2$ & $68.4 \pm 19.8$ & $70.4 \pm 22.4$ & $70.4 \pm 21.0$ \\
GPT (U/L) & $25.2 \pm 1.3$ & $19.8 \pm 0.9$ & $22.4 \pm 1.7$ & $21.6 \pm 0.7$ \\
\hline
\end{tabular}

Nor, normal control; Model, OVA/LPS-induced asthma control; FEMP50, OVA/LPS-induced asthmatic mice treated with $50 \mathrm{mg} / \mathrm{kg} /$ day FEMP; FEMP100, OVA/LPS-induced asthmatic mice treated with $100 \mathrm{mg} / \mathrm{kg} /$ day FEMP. The data are expressed as the mean $\pm \mathrm{SEM}$ levels of $\operatorname{IgE}$ and IL-4 were elevated after the inhalation of OVA/LPS; however, the secretion of IL-17 after stimulation was not higher than that obtained with the normal group, and overall, the secretion levels of IgE, IL-4, and IL-17 in the FEMP-treated groups were lower than those in the model group. The observed decreases in the IgE and IL-4 levels suggest that the FEMP might exert an antiasthma effect by reducing these factors. Consequently, the reduction of IgE and IL-4 production obtained with the FEMP could alleviate various symptoms of asthma. No significant differences in IL-17 secretion in the BALF and serum was found between the normal and model groups. Although the IL-17 level in the BALF of the FEMP100 group was significantly lower than that obtained with the model group, the secretion of IL-17 in the BALF and serum generally did not show a significant difference among the groups. In this study, severe asthma was not induced by OVA and LPS, which might explain why the secretion of IL-17 was not significantly stimulated in the model group compared with the normal group; consequently, no significant decrease in the IL-17 levels in the BALF was found in the FEMP-treated groups with the exception of the FEMP100 group. Therefore, further studies are needed to evaluate the anti-severe asthma effect of the FEMP.

\section{Reducing inflammatory cells and alleviation of lung inflammation by FEMP in OVA/LPS-induced asthma mice model}

After asthma is induced, inflammatory cells, such as eosinophils, neutrophils, and macrophages, usually infiltrate in the lung, and the morphology of the airway smooth muscle layer in the lung becomes thickened compared with that of the normal lung (Ichinose, 2009). As lung inflammation progresses, peribranchial infiltration and alveolar involvement become severe (Dubin and Kolls, 2007). Lung inflammation was observed around bronchial tubes (Fig. 4A), and the lung inflammation score of the model group was also significantly higher than that of the normal group (Fig. 4B). In contrast, the lung inflammation scores of the FEMP-administered groups significantly decreased compared with the model group in a dose dependent manner. The BALF was used to investigate the infiltration of inflammatory cells. The number of total cells in the
BALF of the model group was significantly higher than that in the BALF of the normal group (Fig. 4C). Compared with those found for the model group, the cell numbers were significantly decreased by FEMP treatment in a dosedependent manner. In addition, compared with those found for the model group, the numbers of macrophages and eosinophils were significantly decreased by FEMP treatment in a dose-dependent manner (Fig. 4D). Asthmatic lung tissue has characteristic features, which generally include increased thickness of the lamina propria. The lamina propria of lung tissue is a thin layer that usually involves the mucosa, connective tissue, and blood vessels, whereas the asthmatic lamina propria is filled with diverse inflammatory cells, such as eosinophils, neutrophils, macrophages, and mast cells; consequently, asthma is associated with a characteristic structure in lung tissue (Pesci et al., 1993). The administration of FEMP significantly alleviated lung tissue inflammation and decreased inflammatory cell infiltration in the BALF; in particular, the number of eosinophils was significantly decreased by the FEMP in a dose-dependent manner, and the histological analysis indicated that this treatment alleviated lung tissue inflammation by reducing inflammatory cells. The results suggest that administration of the FEMP significantly inhibits the secretion of inflammatory cytokines, which decreases inflammatory cell infiltration in the BALF and thereby induces the remodeling of lung tissue damaged by inflammation.

\section{Suppression of cough by FEMP in citric acid (CA)- induced coughing guinea pig model}

The cough reflex is essential under physiological conditions and is considered an ordinary symptom of respiratory diseases, such as chronic obstructive pulmonary disease. Chronic cough not only negatively impacts health-related quality of life but might induce an increase in inflammatory mediators by imposing physical stress on airway cells (Wex and Bouyssou, 2015). Cough is also a symptom of asthma. Aggravated inflammatory conditions in the airway stimulate sensory nerves, and this stimulation cause coughing. Chronic cough is caused by cough reflex hypersensitivity, which is generated by mast cell activation and neurogenic inflammation (Qiu et al., 2011). In this 

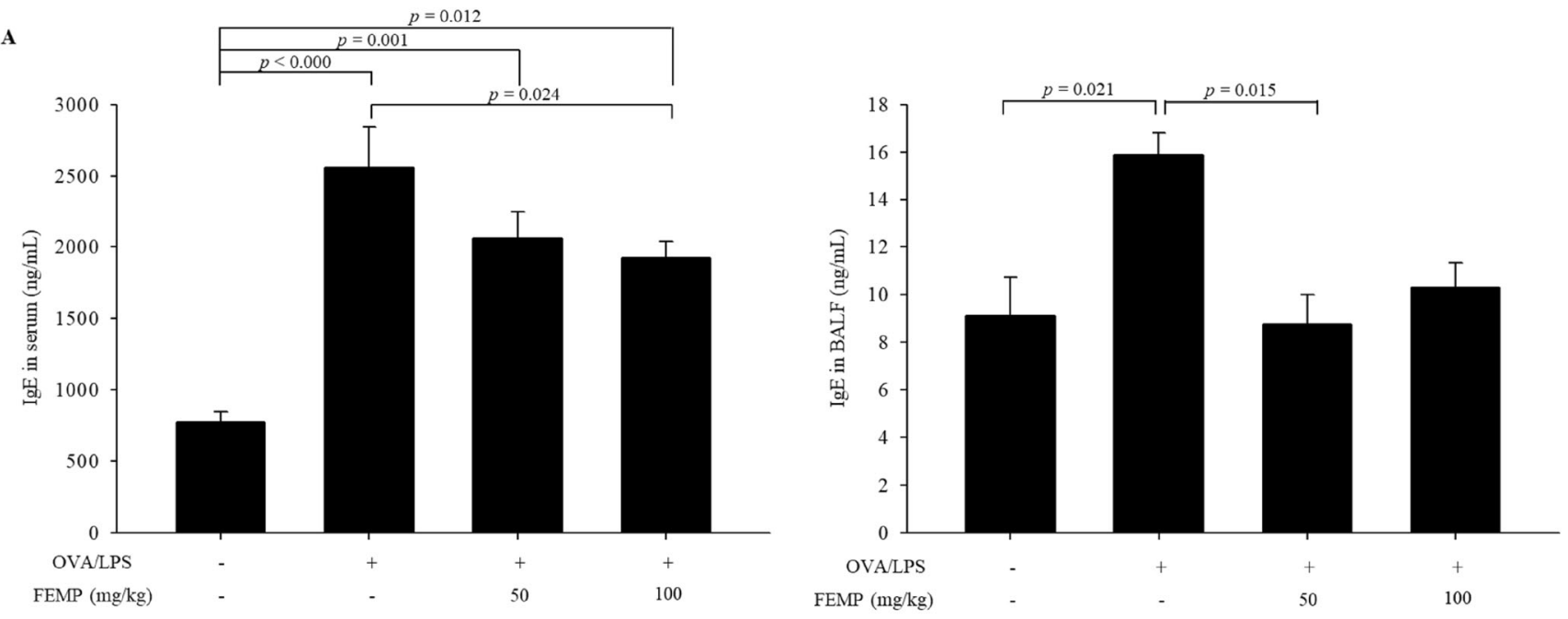

B
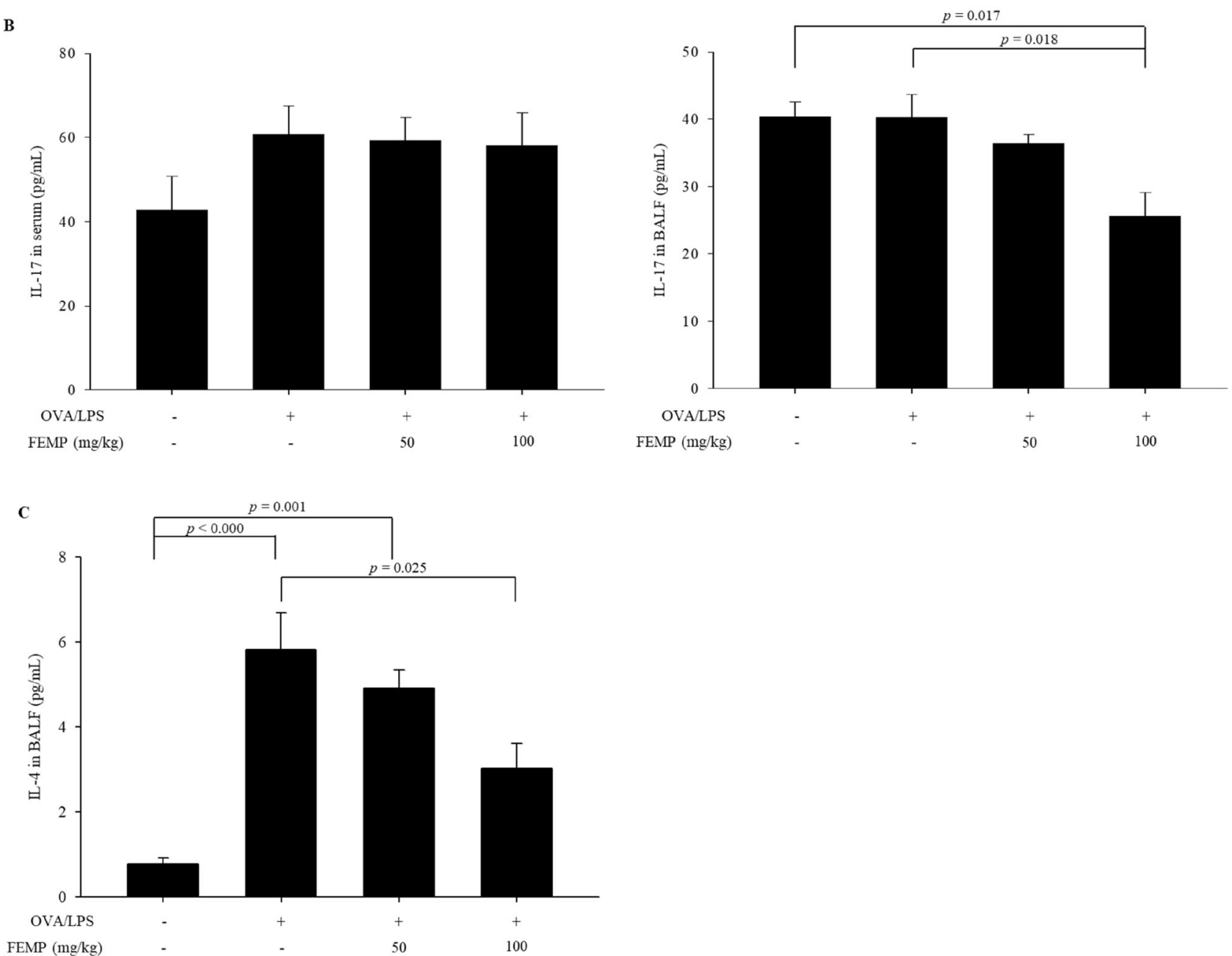

Fig. 3 The FEMP decreased the secretion levels of IgE and IL-4 in an OVA/LPS-induced asthma mouse model ( $n=8 /$ group). BALB/c mice exhibited asthma induced by OVA/LPS, and the levels of IgE and cytokines were measured by ELISA. Secretion level of IgE in the

BALF and serum (A); secretion level of IL-17 in the BALF and serum (B); secretion level of IL-4 in the BALF $(\mathbf{C})$. The data are expressed as the mean \pm SEM and were analyzed by one-way ANOVA/ Tukey's HSD method 

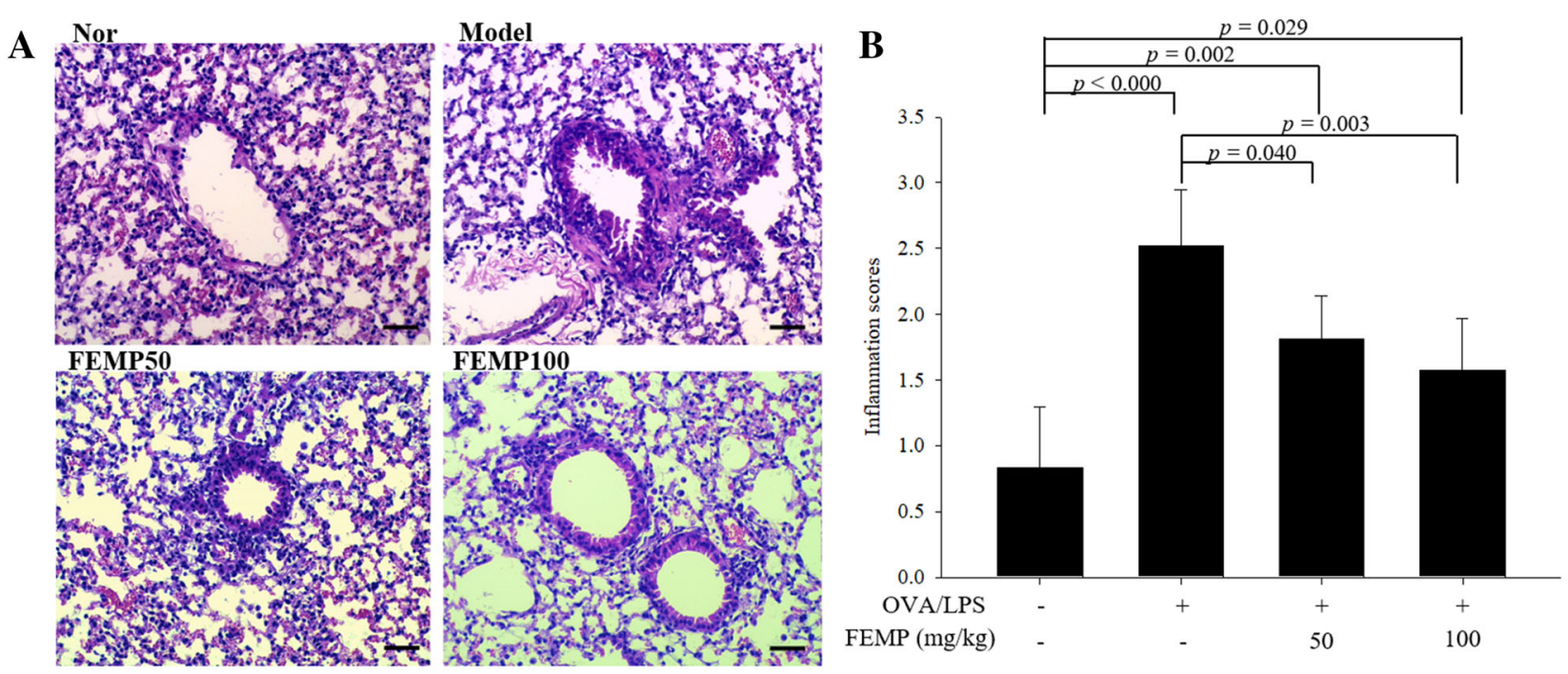

$\mathbf{C}$

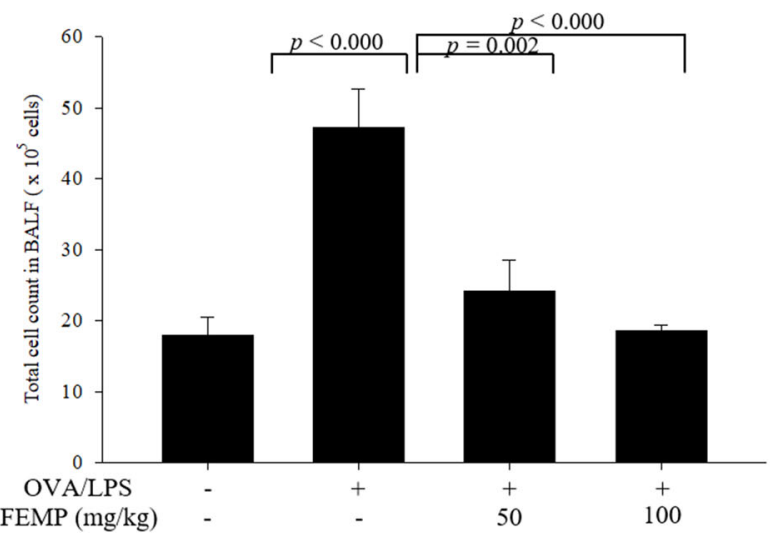

Fig. 4 The FEMP relieved lung tissue inflammation and inflammatory cell infiltration in mice with OVA/LPS-induced asthma ( $n=8$ /group). H\&E staining of lung tissues of mice with OVA/ LPS-induced asthma (A). The scale bar indicates $50 \mu \mathrm{m}$, and the tissue was observed at $200 \times$ magnification. Lung inflammation scores (B); total number of inflammatory cells $(\mathbf{C})$; numbers of

study, a CA-induced coughing model was used to investigate the antitussive effect of the FEMP. CA has been commonly used as a tussive material in animal studies and stimulates $\mathrm{A} \delta$-fiber, $\mathrm{C}$-fiber and A-fiber terminals to induce coughing (Takahama et al., 1993). The active components oxyresveratrol and rosmarinic acid in FEMP have influential anti-inflammatory and antioxidant effects. Antioxidants inhibit cough hypersensitivity induced by hyperoxia (Brozmanova and Hanacek, 2018). Compared with that in the CA-induced control group, the number of CA-induced coughs was significantly decreased by FEMP treatment in a dose-dependent manner (Fig. 5). These results show that the FEMP effectively inhibits CA-induced cough. To examine the effects of FEMP on CA-induced coughing guinea pigs in terms of their body weight or hepatotoxicity, we measured the body weights of the animals and major

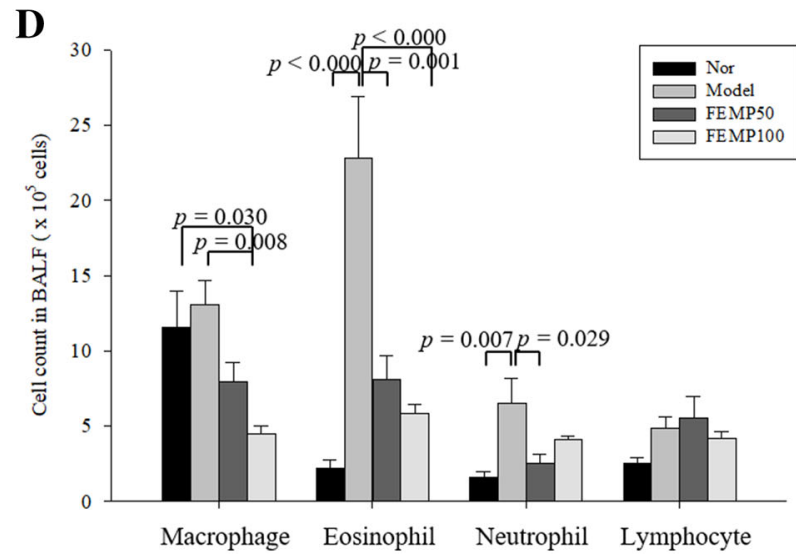

macrophages, eosinophils, neutrophils and lymphocytes (D). Nor, normal control; Model, OVA/LPS-induced asthma control; FEMP50, OVA/LPS-induced asthmatic mice treated with $50 \mathrm{mg} / \mathrm{kg} /$ day FEMP; FEMP100, OVA/LPS-induced asthmatic mice treated with $100 \mathrm{mg} /$ $\mathrm{kg} /$ day FEMP. The data are expressed as the mean \pm SEM and were analyzed by one-way ANOVA/Tukey's HSD method

hepatotoxicity indicators, such as GOT and GPT. Administration of the FEMP did not result in any difference in body weight, and the GOT and GPT measurements indicated no hepatotoxicity (Table S2). These results suggest that the FEMP might protect respiratory health without inducing hepatotoxicity. Although CA-induced coughing does not occur through the asthmatic pathway, the results suggest that the FEMP may inhibit asthma-induced coughing by reducing the secretion of inflammatory cytokines.

Fermentation uses enzymes in microbes to degrade high molecular weight compounds into low molecular weight ones and induces bioconversion, which increases extraction yield and produces new compounds. It converts the compounds that are difficult to absorb into absorbable forms. It also undergoes biochemical reactions to make more 


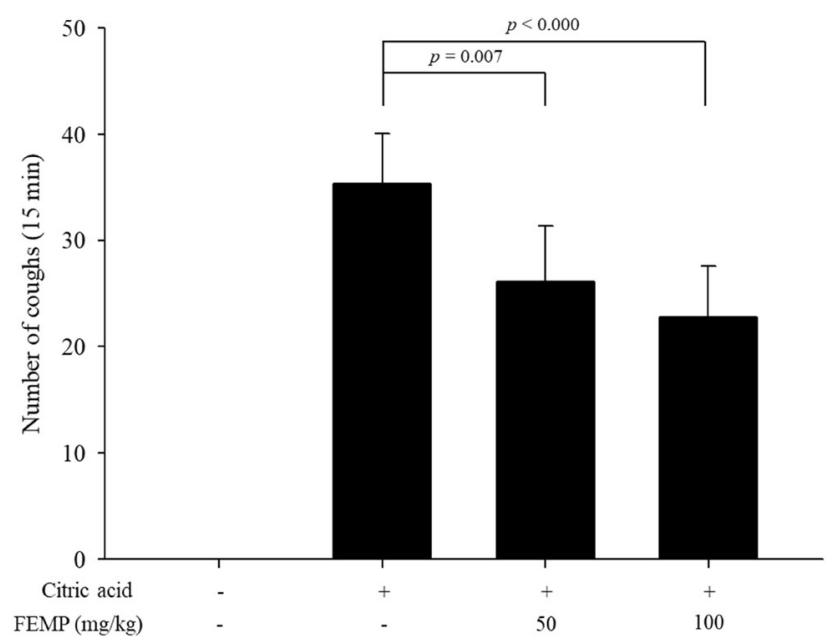

Fig. 5 The FEMP reduced the cough reflex sensitivity in CA-induced coughing guinea pigs ( $\mathrm{n}=8$ /group). The FEMP was administered for one week. Coughing was induced by exposure to $0.2 \mathrm{M}$ citric acid for $10 \mathrm{~min}$, and the number of coughs over the subsequent $15 \mathrm{~min}$ period was counted. The data are expressed as the mean \pm SEM and were analyze by one-way ANOVA/Tukey's HSD method

effective compounds from less effective compounds. Mulberroside A, a stilbene glycoside in the Morus alba $L$. is converted to oxyresveratrol by removing glycoside through bio-conversion, which shows more beneficial activities, for instance, more antioxidative and anti-inflammatory activities (Chung et al., 2003). Rosmarinic acid, an effective component of S. plebeia R. Brown, can be produced by bioconversion from L-phenylalanine and L-tyrosine using Saccharomyces cerevisiae (Babaei et al., 2020). In our further study, we will analyze the chemical composition of FEMP. Oxyresveratrol is a polyphenolic molecule and exerts anti-inflammatory effects by inhibiting the phosphorylation of PI3K/AKT and suppressing NF- KB signaling pathway (Hankittichai et al., 2020). Rosmarinic acids is also a potential anti-inflammatory agent and is known as a strong antioxidant (Colica et al., 2018). Asthma is a complicated disease occurred by diverse causes such as environmental factors, genetic factors, and hygiene hypothesis. Ultimately, these causes induce long-term inflammation in the airways. Especially, oxidative stress induced inflammatory responses are the main cause of exacerbation of asthma pathogenesis (Mishra et al., 2018). Therefore, the anti-inflammatory mediators and antioxidants can have a therapeutic effect to asthma.

In conclusion, fine dust, which causes respiratory diseases, including asthma, has become an important issue worldwide. The fermented water extract of a mixture of medicinal plants, including Ramulus mori, Salvia plebeia R. Brown, and Anthriscus sylvestris, improved asthma and tussive symptoms through its anti-inflammatory activity. In this study, the FEMP exerted anti-inflammatory effects on a lung epithelium cell line and lung tissue from an asthma mouse model through the inhibition of proinflammatory cytokines and exhibited antitussive activity in guinea pigs with CA-induced cough. Moreover, the FEMP did not exhibit cytotoxicity or hepatotoxicity in vitro and in vivo, respectively. Therefore, the FEMP could be used as a potential material for inclusion in functional foods aiming to improve respiratory health.

Supplementary Information The online version contains supplementary material available at https://doi.org/10.1007/s10068021-00955-3.

Acknowledgements This work was supported by the Technology Development Program (S2657856) funded by the Ministry of SMEs and Startups (MSS, Korea).

\section{Declarations}

Conflict of interest The authors declare no conflict of interest.

\section{References}

Ashraf MI, Shahzad M, Shabbir A. Oxyresveratrol ameliorates allergic airway inflammation via attenuation of IL-4, IL-5, and IL-13 expression levels. Cytokine 76: 375-381 (2015)

Babaei M, Borja Zamfir GM, Chen X, Christensen HB, Kristensen M, Nielsen J , Borodina I. Metabolic engineering of Saccharomyces cerevisiae for rosmarinic acid production. ACS Synthetic Biology 9: 1978-1988 (2020)

Bayes H, Ritchie N, Irvine S, Evans T. A murine model of early Pseudomonas aeruginosa lung disease with transition to chronic infection. Scientific Reports 6: 35838 (2016)

Berry M, Brightling C, Pavord I ,Wardlaw AJ. TNF- $\alpha$ in asthma. Current Opinion in Pharmacology 7: 279-282 (2007)

Brightling C, McKenna S, Hargadon B, Birring S, Green R, Siva R, Berry M, Parker D, Monteiro W, Pavord I. Sputum eosinophilia and the short term response to inhaled mometasone in chronic obstructive pulmonary disease. Thorax 60: 193-198 (2005)

Brown JS, Gordon T, Price O, Asgharian B. Thoracic and respirable particle definitions for human health risk assessment. Particle and Fibre Toxicology 10: 12 (2013)

Brozmanova M ,Hanacek J. Hyperoxia-induced regulation of cough reflex and its effect after antioxidant supplementation. Respiratory Physiology \& Neurobiology 257: 75-81 (2018)

Chung KO, Kim BY, Lee MH, Kim YR, Chung HY, Park JH, Moon JO. In-vitro and in-vivo anti-inflammatory effect of oxyresveratrol from Morus alba L. Journal of Pharmacy and Pharmacology 55: 1695-1700 (2003)

Colica C, Di Renzo L, Aiello V, De Lorenzo A, Abenavoli L. Rosmarinic acid as potential anti-inflammatory agent. Reviews on Recent Clinical Trials 13: 240-242 (2018)

Duan W, Chan JH, Wong CH, Leung BP, Wong WF. Antiinflammatory effects of mitogen-activated protein kinase kinase inhibitor U0126 in an asthma mouse model. The Journal of Immunology 172: 7053-7059 (2004)

Dubin PJ, Kolls JK. IL-23 mediates inflammatory responses to mucoid Pseudomonas aeruginosa lung infection in mice. American Journal of Physiology-Lung Cellular and Molecular Physiology 292: L519-L528 (2007) 
Fejes S, Blázovics A, Lugasi A, Lemberkovics É, Petri G, Kéry Á. In vitro antioxidant activity of Anthriscus cerefolium L.(Hoffm.) extracts. Journal of Ethnopharmacology 69: 259-265 (2000)

Gould HJ, Sutton BJ, Beavil AJ, Beavil RL, McCloskey N, Coker HA, Fear D, Smurthwaite L. The biology of IGE and the basis of allergic disease. Annual Review of Immunology 21: 579-628 (2003)

Guimarães R, Barros L, Carvalho AM, Ferreira IC. Infusions and decoctions of mixed herbs used in folk medicine: synergism in antioxidant potential. Phytotherapy Research 25: 1209-1214 (2011)

Hankittichai P, Lou HJ, Wikan N, Smith DR, Potikanond S, Nimlamool W. Oxyresveratrol inhibits IL-1 $\beta$-induced inflammation via suppressing AKT and ERK1/2 activation in human microglia, HMC3. International Journal of Molecular Sciences 21: $6054(2020)$

Harley RM, Atkins S, Budantsev AL, Cantino PD, Conn BJ, Grayer R, Harley MM, De Kok Rd, Krestovskaja Td, Morales R, Paton AJ, Ryding O, Upson T. Labiatae. vol. 7, pp. 167-275. In: Flowering Plants. Dicotyledons. Kadereit JW (ed). SpringerVerlag Berlin Heidelberg Heidelberg, New York, NY, USA (2004)

Hedges JC, Singer CA, Gerthoffer WT. Mitogen-activated protein kinases regulate cytokine gene expression in human airway myocytes. American Journal of Respiratory Cell and Molecular Biology 23: 86-94 (2000)

Heuer M, Behlich A-S, Lee J-S, Ribechini E, Jo E-K, Lutz MB. The 30-kDa and 38-kDa antigens from Mycobacterium tuberculosis induce partial maturation of human dendritic cells shifting CD4+ $\mathrm{T}$ cell responses towards IL-4 production. BMC Immunology 14: 48 (2013)

Ichinose M. Differences of inflammatory mechanisms in asthma and COPD. Allergology International 58: 307-313 (2009)

Jo SY, Lee UY, Kim EY, Lee SJ, Her JW, Yoon TJ. A study on the anti-inflammatory and anti-allergic effect of Salvia plebeia R. extracts. Korean Journal of Pharmacognosy 41: 31-37 (2010)

Kolls JK, Lindén A. Interleukin-17 family members and inflammation. Immunity 21: 467-476 (2004)

Lambrecht BN, Hammad H ,Fahy JV. The cytokines of asthma. Immunity 50: 975-991 (2019)

Leach C, Davidson P, Boudreau R. Improved airway targeting with the CFC-free HFA-beclomethasone metered-dose inhaler compared with CFC-beclomethasone. European Respiratory Journal 12: 1346-1353 (1998)

Livak KJ, Schmittgen TD. Analysis of relative gene expression data using real-time quantitative PCR and the $2-\Delta \Delta \mathrm{CT}$ method. Methods 25: 402-408 (2001)

Mishra V, Banga J, Silveyra P. Oxidative stress and cellular pathways of asthma and inflammation: Therapeutic strategies and pharmacological targets. Pharmacology \& Therapeutics 181: 169-182 (2018)

Pesci A, Foresi A, Bertorelli G, Chetta A, Olivieri D, Oliveri D. Histochemical characteristics and degranulation of mast cells in epithelium and lamina propria of bronchial biopsies from asthmatic and normal subjects. American Review of Respiratory Disease 147: 684-689 (1993)

Phillipps G. Structure-activity relationships of topically active steroids: the selection of fluticasone propionate. Respiratory Medicine 84: 19-23 (1990)

Qiu Z, Yu L, Xu S, Liu B, Zhao T, Lue H, Qiu Z. Cough reflex sensitivity and airway inflammation in patients with chronic cough due to non-acid gastro-oesophageal reflux. Respirology 16: 645-652 (2011)

Ramakrishnan RK, Al Heialy S, Hamid Q. Role of IL-17 in asthma pathogenesis and its implications for the clinic. Expert Review of Respiratory Medicine 13: 1057-1068 (2019)

Sanbongi C, Takano H, Osakabe N, Sasa N, Natsume M, Yanagisawa $\mathrm{R}$, Inoue Ki, Sadakane K, Ichinose T, Yoshikawa T. Rosmarinic acid in perilla extract inhibits allergic inflammation induced by mite allergen, in a mouse model. Clinical \& Experimental Allergy 34: 971-977 (2004)

Sokol CL, Barton GM, Farr AG ,Medzhitov R. A mechanism for the initiation of allergen-induced $\mathrm{T}$ helper type 2 responses. Nature Immunology 9: 310-318 (2008)

Steinke JW, Borish L. Th2 cytokines and asthma-Interleukin-4: its role in the pathogenesis of asthma, and targeting it for asthma treatment with interleukin-4 receptor antagonists. Respiratory Research 2: 66-70 (2001)

Takahama K, Fuchikami J, Suzuki A, Tabata T, Kai H, Miyata T. Differences in the mode of cough augmentation by four angiotensin-converting enzyme inhibitors in guinea-pigs. Journal of Pharmacy and Pharmacology 45: 1003-1005 (1993)

Wex E, Bouyssou T. Olodaterol attenuates citric acid-induced cough in naïve and ovalbumin-sensitized and challenged guinea pigs. PLoS One 10: e0119953 (2015)

Wong C, Ho C, Ko F, Chan C, Ho A, Hui D, Lam C. Proinflammatory cytokines (IL-17, IL-6, IL-18 and IL-12) and Th cytokines (IFN$\gamma$, IL-4, IL-10 and IL-13) in patients with allergic asthma. Clinical \& Experimental Immunology 125: 177-183 (2001)

Wu J-Z, Ge D-D, Zhou L-F, Hou L-Y, Zhou Y, Li Q-Y. Effects of particulate matter on allergic respiratory diseases. Chronic Diseases and Translational Medicine 4: 95-102 (2018)

Yu W, Chen H, Xiang Z, He N. Preparation of polysaccharides from Ramulus mori, and their antioxidant, anti-inflammatory and antibacterial activities. Molecules 24: 856 (2019)

Zhang L-L, Chen B, Fan X-Y, Wu S-S, Zhang S-Q, Wu H-M. LPS cooperates with poly-L-arginine to promote IL-6 and IL-8 release via the JNK signaling pathway in NCI-H292 cells. Journal of Immunology Research 2016: 3421060 (2016)

Zhang Z, Shi L. Anti-inflammatory and analgesic properties of cismulberroside A from Ramulus mori. Fitoterapia 81: 214-218 (2010)

Publisher's Note Springer Nature remains neutral with regard to jurisdictional claims in published maps and institutional affiliations. 